\title{
The effect of geothermal water on skinfold and body circumferences
}

\author{
LOLITA RAPOLIENE் $\dot{A}^{\mathrm{A}-\mathrm{G}}$, AELITA SKARBALIENE் ${ }^{\mathrm{B}, \mathrm{E}}$, LINA GEDRIME் ${ }^{\mathrm{C}-\mathrm{G}}$ \\ ORCID ID: 0000-0002-5782-0937
}

DAIVA MOCKEVIČIENÉ ${ }^{B}$, SAMANTA KAZLAUSKAITÉE, F

Faculty of Health Sciences, Klaipeda University, Lithuania

A - Study Design, B - Data Collection, C - Statistical Analysis, D - Data Interpretation, E - Manuscript Preparation, F - Literature Search, G - Funds Collection

Summary Background. Balneotherapy is one of the basic methods of treatment widely used in natural medicine.

Objectives. To investigate the effect of geothermal water on body mass index, body skinfolds and circumferences and serum cholesterol.

Material and methods. A randomised, controlled, single-blinded follow-up interventional study was performed on 204 workers divided into 5 groups: balneotherapy with 20,40 and $60 \mathrm{~g} / /$ total mineralisation, tap water and a control group. A 20-minute bath treatment was carried out on an everyday basis for 5 days a week over a 2-week period. Body circumferences and skinfolds, body mass index and cholesterol were measured before and after the treatment.

Results. The best effect reducing body volume after 2 weeks was obtained in the $20 \mathrm{~g} / \mathrm{group}(7.5 \mathrm{~cm})$, but the biggest and longest effect was achieved in the $60 \mathrm{~g} / \mathrm{I}$ group $(8.4 \mathrm{~cm}$ from baseline). The greatest and longest-lasting decrease in skinfold was after $60 \mathrm{~g} / \mathrm{l}$ procedures (22.4 mm from baseline). Significant positive changes for total cholesterol were seen after 20 (MD 0.2) and $40 \mathrm{~g} / \mathrm{l}$ (MD 0.3) baths, for low-density lipoprotein after $40 \mathrm{~g} / \mathrm{I}$ (MD 0.1) baths, and for triglyceride levels after $20 \mathrm{~g} / \mathrm{l}$ baths. There were no significant changes in all groups regarding body mass index.

Conclusions. 20-minute baths of geothermal water are more effective than tap water for lowering of hypodermic fat content in the body and cholesterol level in serum. $60 \mathrm{~g} / \mathrm{l}$ baths have a long-lasting effect on body fat volumes, and $20-40 \mathrm{~g} / \mathrm{l}$ geothermal water is appropriate for an antilipidemic effect.

Key words: cholesterol, balneology, adipose tissue.

Rapolienè L, Skarbalienè A, Gedrimè L, Mockevičienė D, Kazlauskaitè S. The effect of geothermal water on skinfold and body circumferences. Fam Med Prim Care Rev 2019; 21(4): 364-371, doi: https://doi.org/10.5114/fmpcr.2019.90170.

\section{Background}

Balneotherapy is the therapeutic use of bathing agents such as mineral and thermal waters and is one of the basic methods of treatment widely used in natural medicine [1]. It involves treating different health problems by bathing, usually in hot springs and other mineral-rich waters. The core effects of balneotherapy are changes caused by the influence of mechanical, thermal and chemical factors through the skin and mucous membranes as a result of the neuroreflexive, humoral mechanisms involved, caused by biochemically active substances $[2,3]$. Thus, balneotherapy includes three key elements: (1) the temperature of water for balneotherapy must be at least 20 degrees Celsius (68 degrees Fahrenheit), although it is often much warmer, at 34 degrees Celsius (93 degrees Fahrenheit); (2) mineral content: balneotherapy requires water containing dissolved materials at a concentration of at least 1 gram per litre. The dissolved substances most often include salts, sulphur compounds or gases; (3) natural occurrence, i.e. natural springs. A spring forms when an aquifer fills to the point that the water overflows onto the surface of the land. They range in size from small seeps to huge pools, and they vary greatly in their mineral content [4]. Balneotherapy is a frequent kind of treatment at spas and health resorts. Scientific literature has shown that health resort treatment is associated with clinical improvement in diseases of the skin, respiratory, circulatory, gastrointestinal and nervous systems, cancer, nutritional and metabolic disorders, mental disorders, diseases of the ear, endocrine diseases, female genital diseases and nutritional deficiencies [5-7]. Balneotherapy is a safe and time-tested treatment, and scientists believe that mineral water treatment methods compared to similar non-mineral treatments have better and longer-lasting improvements concerning pain, function, quality of life, clinical parameters and others [3].

$39 \%$ of adults aged 18 years and over were overweight in 2016 , and $13 \%$ were obese. Thus, worldwide obesity has nearly tripled since 1975. Statistics reveal that 41 million children under the age of 5 were overweight or obese in 2016, and over 340 million children and adolescents aged 5-19 were overweight or obese in 2016. Overweight and obesity are the main risk factors in coronary heart disease, strokes, type II diabetes, osteoarthritis, certain types of cancer (uterus, breast, intestine) and sleep quality, and they thereby worsen the quality of life, increase mortality and add to health-related costs [8,9]. Serum cholesterol fractions most frequently correlate with overweight and are a prognostic factor for cardiovascular diseases. Scientists found that balneotherapy induces cardiac activity, blood flow, temperature changes in the body, increase interstitial fluid transfer into the vascular space, accelerates the removal of metabolites and waste products and enhances delivery of oxygen [10], which has an impact on the plasma level of the adipocytokines leptin and adiponectin [11]. Some scientific studies on humans showed a reduction in excessive body mass and correction of blood lipid spectrum [12], but there is still a lack of controlled studies for body volume. 


\section{Objectives}

The research showed that obesity is preventable. Thus, the aim of our study was to investigate the effect of geothermal water on body mass index (BMI), body skinfolds and circumferences.

\section{Material and methods}

\section{Trial design}

A randomised, controlled, single-blinded interventional study was performed.

\section{Participants}

Data was collected from 250 participants during the period of May-September 2018 in Klaipeda, Lithuania. Hydrotherapy procedures were carried out at the Rehabilitation Department of Klaipeda Seamen's Health Care Centre. The evaluation of participants' clinical statement at baseline and after 2 weeks of treatment was made at the Klaipeda Science and Technology Park facility - Business incubator. An interventional study was implemented in observance of the rules of good clinical practice. Inclusion criteria: current workers aged 18-65 years with no current disease and no rehabilitation procedures during the past 3 months, at least 2 symptoms of distress or a symptom intensity of more than 2 according to the general symptom distress scale (GSDS, T.A. Badger). Exclusion criteria were as follows: acute neurological deficit, epilepsy, inflammatory condition, cutaneous lesion, failure of respiratory/cardiovascular systems, kidney failure, unstable metabolic disorders, severe arrhythmia, febrile infections, bleeding, pregnancy.

Participants were randomly divided into 5 groups of 50 individuals per group: 3 groups of baths of water of different salinity (20, 40, $60 \mathrm{~g} /$ / total salinity water), 1 group of pure water baths, 1 control group (without treatment). All subjects were informed about the purpose, conditions and course of the study prior to inclusion and signed a participant's agreement. The participants of the control group were not given any therapy.

\section{Intervention}

Bath treatment (geothermal or tap water) was carried out on an outpatient on an everyday basis, for 5 days a week over a 2-week period (10 procedures). The geothermal water used was highly mineralised (108 g/l) Na-Cl-Ca-Mg-SO $\mathrm{A}^{\prime} \mathrm{pH}$ 6.07) from the Geoterma 2P (ID 25871) borehole (1,135 m depth). Individual hydrotherapy procedures were as follows: in accordance with calculations of dilution baths ( $400 \mathrm{I}$ ) were prepared as follows: $20 \mathrm{~g} / \mathrm{l}(2 \%)$ bath was filled with 73 I of geothermal water and 327 I of pure tap water; $40 \mathrm{~g} / \mathrm{I}(4 \%)-145$ I geothermal and 254 I of pure tap water; $60 \mathrm{~g} / \mathrm{I}(6 \%)-218$ I geothermal and 181 I pure tap water (additionally warmed in special heaters). The temperature of the baths was $36^{\circ} \mathrm{C}$. The participants had baths (immersion up to the armpits) monitored by trained personnel. Each participant was told to move slightly in the bathtub [13]. The geothermal water chemical composition in the balneotherapy group is shown in Table 1.

\begin{tabular}{|l|l|l|l|}
\hline \multicolumn{5}{|c|}{ Table 1. Mineral composition of geothermal water in the groups } \\
\hline Element, mg/l & I group & II group & III group \\
\hline $\mathrm{Cl}$ & 17,110 & 25,130 & 38,400 \\
\hline $\mathrm{SO}_{4}$ & 526 & 735 & 1,160 \\
\hline $\mathrm{HCO}_{3}$ & 190 & 161 & 125 \\
\hline $\mathrm{CO}_{3}$ & 0,06 & 0.05 & 0.01 \\
\hline $\mathrm{Na}$ & 7,124 & 10,550 & 16,500 \\
\hline $\mathrm{K}$ & 181 & 255 & 428 \\
\hline
\end{tabular}

\begin{tabular}{|l|l|l|l|}
\hline $\mathrm{Ca}$ & 2,500 & 3,550 & 5,110 \\
\hline $\mathrm{Mg}$ & 659 & 940 & 1,430 \\
\hline $\mathrm{Fe}$ & $<0.01$ & $<0.01$ & 0.04 \\
\hline $\mathrm{pH}$ & 7.28 & 7.27 & 6.72 \\
\hline
\end{tabular}

\section{Study outcome}

Body fat parameters changed after balneotherapy with geothermal water of different salinity in comparison with tap water and no treatment. BMI, serum cholesterol levels and body circumferences, and skinfolds were measured before the treatment, after 2-week treatment and 3 month after the treatment. The body circumferences of the upper arm (tense, relaxed), waist, hip, thigh and calf were measured using measuring tape $(\mathrm{cm})$; the skinfolds (biceps, abdomin, triceps, subscapular suprailiac, quadriceps, calf) were measured using calliper testing. Two measurements were taken at each site following standard procedures [14, 15].

\section{Statistical procedures}

Data was reported as the mean \pm SD for continuous variables, or as frequencies in the case of categorical variables. Descriptive statistics and univariate analyses were carried out using SPSS V23.0 (SPSS Inc., Chicago, Illinois, USA). Pearson $\chi^{2}$ tests and independent-sample $t$-tests were used to compare the independent variables versus dependent variables. The hypothesis about the equality of probability distribution was checked against the Mann-Whitney-Wilcoxon $U$ non-parametric criterion, and the corresponding $95 \% \mathrm{Cls}$ were calculated. The Wilcoxon Sign and Kruscal-Wallis non-parametric tests were also used, and a $p$-value $<0.05$ was considered to be significant for all tests.

\section{Ethical consideration}

The study was carried out with the authorisation of the Kaunas Regional Biomedical Research Ethics Committee (permission No. BE-2-1).

\section{Results}

\section{Participant flow}

The flow diagram of the study process is shown in Figure 1.

\section{Recruitment}

The data was collected during the period of May-September 2018 in Klaipeda, Lithuania (primary measurement and 3-month follow-up period).

\section{Baseline data and numbers analysed}

The main characteristics of the survey participants are in Table 2. The study groups did not differ in gender, marital status and physical activity. Participants of the $60 \mathrm{~g} / \mathrm{l}$ geothermal and control groups were older than in other groups; participants in the control group had the biggest BMI.

\section{Outcomes and estimation}

$\mathrm{BMI}$ changes in the groups are showed in Table 3. Despite positive BMI changes in the 20 and $60 \mathrm{~g} / \mathrm{l}$ groups, there were no significant changes in all groups.

The best effect reducing body volume after 2 weeks was obtained in the $20 \mathrm{~g} / \mathrm{l}$ group (Figure 2). Positive significant changes were seen in all measuring points, with the biggest difference in the thigh and hip. $40 \mathrm{~g} / \mathrm{l}$ procedures were effective for $25 \%, 60 \mathrm{~g} / \mathrm{l}-$ for $88 \%$ of body circumferences. Tap water and no treatment in $75 \%$ and $100 \%$ of cases produced a negative effect. The largest volume lost was achieved after $20 \mathrm{~g} / \mathrm{l}$ procedures $(7.5 \mathrm{~cm})$, less - after 60 $\mathrm{g} / \mathrm{l}$ procedures $(5.2 \mathrm{~cm})$. An overall increase in volumes of $3.8 \mathrm{~cm}$ was seen in the tap water group, with $4.4 \mathrm{~cm}$ in the control group. 


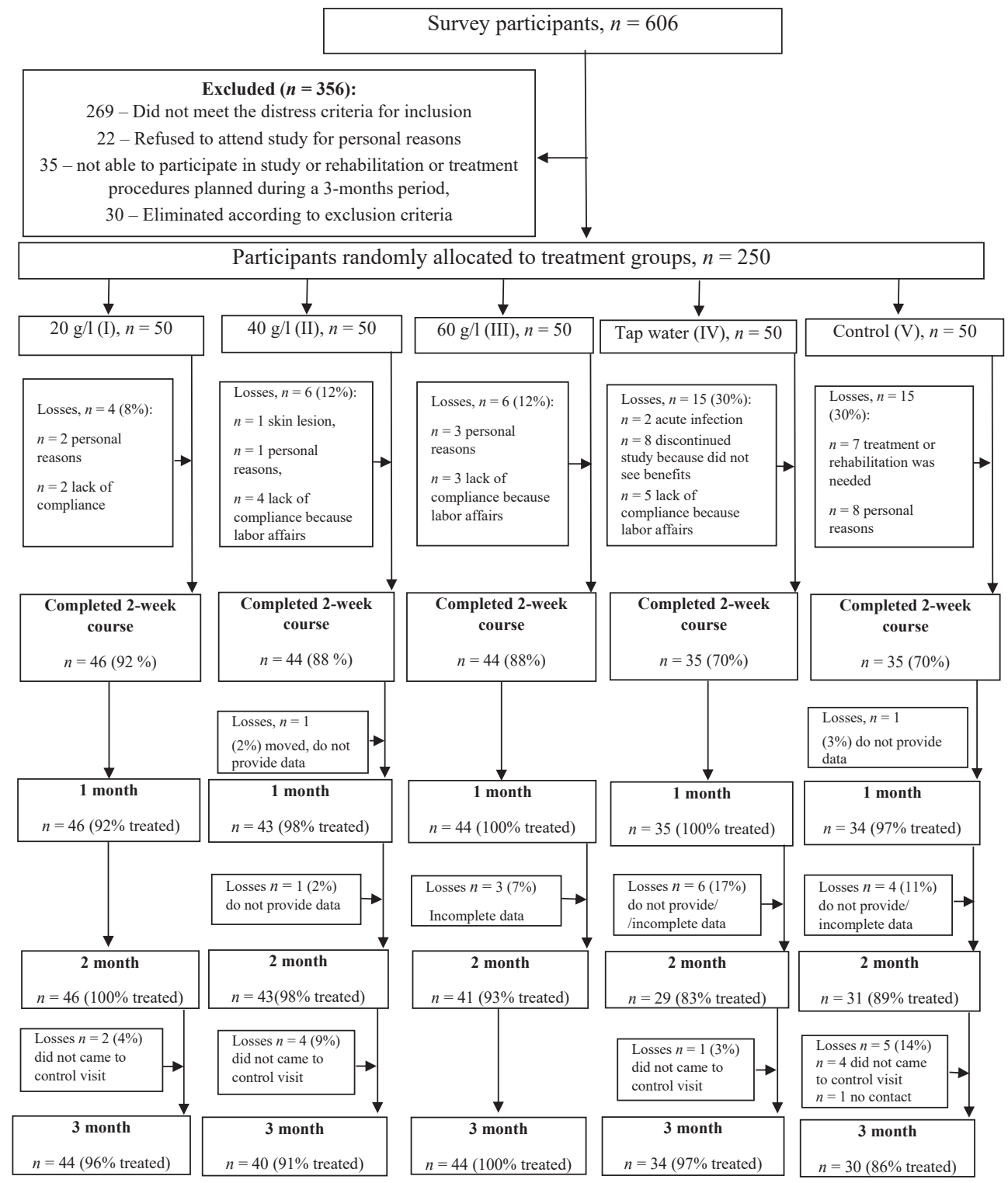

Figure 1. Disposition of the study participants

\begin{tabular}{|c|c|c|c|c|c|c|}
\hline & $I(n=46)$ & II $(n=44)$ & III $(n=44)$ & IV $(n=35)$ & $\mathrm{V}(n=35)$ & $p$ \\
\hline Age, $n(\%)$ & $39.7(10.5)$ & $42.6(10.4)$ & $47.7(9.5)$ & $42.8(12.8)$ & $48.1(12.0)$ & 0.009 \\
\hline \multicolumn{6}{|l|}{ Gender, $n(\%)$} & \multirow{3}{*}{0.638} \\
\hline Men & $4(8.7)$ & $7(15.9)$ & 7 (15.9) & $4(11.4)$ & $7(20)$ & \\
\hline Women & $42(91.3)$ & 37 (84.1) & $37(84.1)$ & 31 (88.6) & $28(80)$ & \\
\hline \multicolumn{6}{|l|}{ Marital status, $n(\%)$} & \multirow{6}{*}{0.073} \\
\hline Prefer not to say & $1(2.2)$ & $2(4,5)$ & & & & \\
\hline Married & $30(65.2)$ & $28(63.6)$ & $32(72.7)$ & $22(62.9)$ & $25(71.4)$ & \\
\hline Single & $11(23.9)$ & $8(18.2)$ & $4(9.1)$ & $7(20)$ & $3(8.6)$ & \\
\hline Divorced & $4(8.7)$ & $6(13.6)$ & $4(9.1)$ & $6(17.1)$ & $3(8.6)$ & \\
\hline Widowed & & & $4(9.1)$ & & $4(11.4)$ & \\
\hline \multicolumn{6}{|c|}{ Level of education, $n$ (\%) } & \multirow{7}{*}{0.004} \\
\hline $\begin{array}{l}\text { Incomplete secondary } \\
\text { education }\end{array}$ & $2(4.3)$ & $1(2.3)$ & & & & \\
\hline Secondary & $4(8.7)$ & $6(13.6)$ & $2(4.5)$ & $6(17.1)$ & 4 (11.4) & \\
\hline Higher & $7(15.2)$ & $3(6.8)$ & $3(6.8)$ & $3(8.6)$ & $11(31.4)$ & \\
\hline High & $1(2.2)$ & $6(13.6)$ & $3(6.8)$ & $2(5.7)$ & $2(5.7)$ & \\
\hline University & $29(63.0)$ & $24(54.5)$ & $30(68.2)$ & $12(34.3)$ & $13(37.1)$ & \\
\hline $\mathrm{PhD}$ & $3(6.5)$ & $4(9.1)$ & $6(13.6)$ & $11(31.4)$ & $5(14.3)$ & \\
\hline
\end{tabular}




\begin{tabular}{|c|c|c|c|c|c|c|}
\hline & $I(n=46)$ & II $(n=44)$ & III $(n=44)$ & IV $(n=35)$ & $\mathrm{V}(n=35)$ & $p$ \\
\hline \multicolumn{6}{|c|}{ Physical activity, $\boldsymbol{n}(\%)$} & \multirow{8}{*}{0.080} \\
\hline Every day & $6(13.0)$ & 7 (15.9) & $5(11.4)$ & $3(8.6)$ & $4(11.4)$ & \\
\hline 4-6 times/week & $1(2.2)$ & $8(18.2)$ & $1(2.3)$ & & $3(8.6)$ & \\
\hline 2-3 times/week & $13(28.3)$ & $11(25)$ & $16(36.4)$ & $13(37.1)$ & $17(48.6)$ & \\
\hline Once per week & $6(13.0)$ & $8(18.2)$ & $4(9.1)$ & 6 (17.1) & $3(8.6)$ & \\
\hline 2-3 times/month & $7(15.2)$ & $3(6.8)$ & $6(13.6)$ & $3(8.6)$ & $2(5.7)$ & \\
\hline Few times/year & $5(10.9)$ & $4(9.1)$ & $5(11.4)$ & $7(20)$ & $4(11.4)$ & \\
\hline Never & 8 (17.4) & $1(2.3)$ & $7(15.9)$ & $3(8.6)$ & $2(5.7)$ & \\
\hline
\end{tabular}

\begin{tabular}{|l|l|l|l|l|l|l|l|}
\hline \multicolumn{2}{|l|}{ Table 3. Changes of BMI in the study groups } \\
\hline & Mean & SD & MD & CI lower & Cl upper & $t$ & $p$ \\
\hline I group & 24.83 & 5.80 & 0.08 & -0.0490 & 0.2012 & 1.225 & 0.227 \\
\hline & 24.75 & 5.76 & & & & & \\
\hline II group & 25.16 & 4.62 & -0.02 & -0.1827 & 0.1463 & -0.223 & 0.825 \\
\hline & 25.18 & 4.71 & & & & & \\
\hline III group & 26.55 & 5.59 & 0.06 & -0.0536 & 0.1718 & 1.058 & 0.296 \\
\hline & 26.49 & 5.46 & & & & & \\
\hline IV group & 25.42 & 3.75 & -0.08 & -0.2202 & 0.0614 & -1.147 & 0.260 \\
\hline & 25.50 & 3.80 & & & & & \\
\hline V group & 29.36 & 7.38 & -0.09 & -0.1927 & 0.0162 & -1.719 & 0.095 \\
\hline & 29.45 & 7.50 & & & & & \\
\hline
\end{tabular}

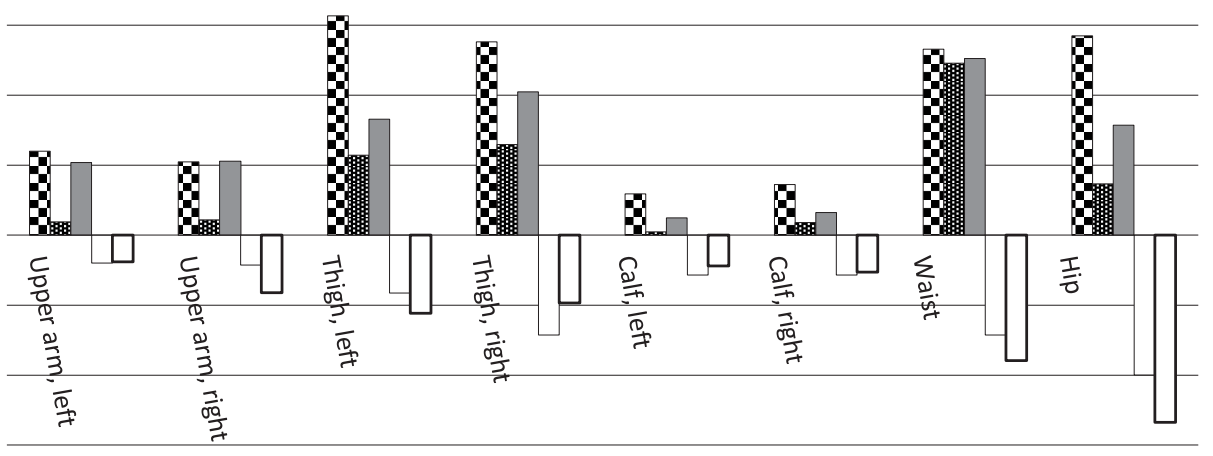

口I 国 II $\square$ III $\square$ IV $\square \mathrm{V}$

Figure 2. Change in body circumferences in the study groups after 2 weeks

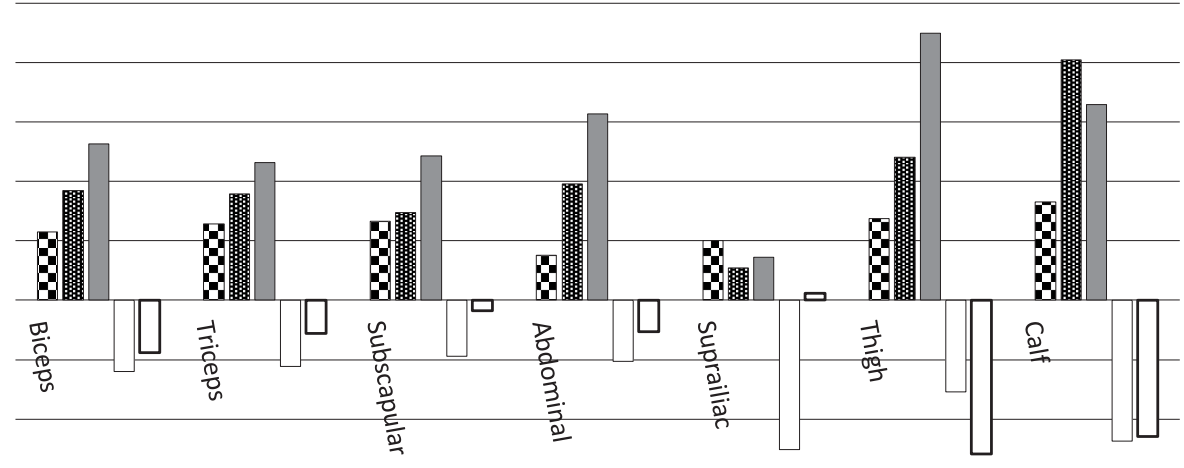

田 俩II $\square$ III $\square$ IV $\square \mathrm{V}$

Figure 3. Change in body skinfolds in the study groups after 2 weeks 
After 3 months, significant positive changes remained in $25 \%$ of the measurements in the $20(2.9 \mathrm{~cm})$ and $40 \mathrm{~g} / \mathrm{l}(1.9$ $\mathrm{cm}$ ) groups, while in the $60 \mathrm{~g} / \mathrm{l}$ group $-63 \%(8.4 \mathrm{~cm}$ from baseline) (Table 4). The tap water and control groups saw a negative change (1.4 and $2.6 \mathrm{~cm}$, respectively).

The study results showed that all skinfolds significantly decreased in the 20 and $60 \mathrm{~g} / \mathrm{l}$ groups, as well as in $86 \%$ of the cases in the $40 \mathrm{~g} / \mathrm{l}$ group. The biggest effect was seen in the 60 $\mathrm{g} / \mathrm{l}$ group $(19 \mathrm{~mm})$, and less in the $40 \mathrm{~g} / \mathrm{l}$ group $(14 \mathrm{~mm})$. The skinfolds increased in the tap water $(10.7 \mathrm{~mm})$ and control $(6.9$ $\mathrm{mm}$ ) groups (Figure 3).

After 3 month, $86 \%$ of skinfolds remained significantly smaller in the 20, 40 and $60 \mathrm{~g} / \mathrm{l}$ geothermal groups from baseline $18,7,16$ and $22,8 \mathrm{~mm}$, respectively. $71 \%$ of skinfolds in the tap water and control groups significantly decreased after 3 months (Table 5).

\begin{tabular}{|c|c|c|c|c|c|c|c|c|c|c|}
\hline & \multicolumn{2}{|l|}{$\mathrm{I}(20 \mathrm{~g} / \mathrm{l})$} & \multicolumn{2}{|l|}{ II $(40 \mathrm{~g} / \mathrm{l})$} & \multicolumn{2}{|l|}{ III $(60 \mathrm{~g} / \mathrm{l})$} & \multicolumn{2}{|l|}{ IV(tap) } & \multicolumn{2}{|l|}{ V (control) } \\
\hline & \begin{tabular}{|l} 
Mean \\
difference \\
$(\mathrm{cm})$
\end{tabular} & $p$ & \begin{tabular}{|l} 
Mean \\
difference \\
$(\mathrm{cm})$
\end{tabular} & $p$ & $\begin{array}{l}\text { Mean } \\
\text { difference } \\
(\mathrm{cm})\end{array}$ & $p$ & $\begin{array}{l}\text { Mean } \\
\text { difference } \\
(\mathrm{cm})\end{array}$ & $p$ & $\begin{array}{l}\text { Mean } \\
\text { difference } \\
(\mathrm{cm})\end{array}$ & $p$ \\
\hline \multicolumn{11}{|c|}{ Upper arm, left } \\
\hline After 2 w & 0.59 & 0.00 & 0.09 & 0.50 & 0.52 & 0.00 & -0.20 & 0.14 & -0.19 & 0.01 \\
\hline $3 \mathrm{mo}$ & -0.11 & 0.42 & -0.067 & 0.70 & 0.48 & 0.03 & -0.20 & 0.10 & -0.48 & 0.00 \\
\hline \multicolumn{11}{|c|}{ Upper arm, right } \\
\hline After $2 \mathrm{w}$ & 0.52 & 0.00 & 0.11 & 0.40 & 0.53 & 0.00 & -0.21 & 0.00 & -0.41 & 0.00 \\
\hline $3 \mathrm{mo}$ & -0.04 & 0.79 & -0.29 & 0.17 & 0.62 & 0.01 & -0.51 & 0.01 & -0.38 & 0.00 \\
\hline \multicolumn{11}{|l|}{ Waist } \\
\hline After $2 \mathrm{w}$ & 1.33 & 0.00 & 1.23 & 0.14 & 1.26 & 0.00 & -0.71 & 0.11 & -0.89 & 0.00 \\
\hline $3 \mathrm{mo}$ & 0.14 & 0.72 & -0.24 & 0.64 & 0.71 & 0.17 & -0.71 & 0.18 & -0.60 & 0.11 \\
\hline \multicolumn{11}{|l|}{ Hips } \\
\hline After 2 w & 1.42 & 0.00 & 0.36 & 0.12 & 0.78 & 0.00 & -1.00 & 0.00 & -1.34 & 0.00 \\
\hline $3 \mathrm{mo}$ & 0.17 & 0.61 & 0.32 & 0.36 & 1.45 & 0.00 & -0.53 & 0.21 & -0.16 & 0.63 \\
\hline \multicolumn{11}{|l|}{ Thigh, left } \\
\hline After 2 w & 1.56 & 0.00 & 0.57 & 0.04 & 0.82 & 0.00 & -0.41 & 0.06 & -0.56 & 0.01 \\
\hline $3 \mathrm{mo}$ & 1.21 & 0.00 & 0.81 & 0.04 & 2.36 & 0.00 & 0.47 & 0.20 & -0.26 & 0.45 \\
\hline \multicolumn{11}{|c|}{ Thigh, right } \\
\hline After $2 \mathrm{w}$ & 1.38 & 0.00 & 0.65 & 0.05 & 1.02 & 0.00 & -0.71 & 0.00 & -0.48 & 0.01 \\
\hline $3 \mathrm{mo}$ & 1.32 & 0.00 & 1.16 & 0.01 & 2.57 & 0.00 & 0.43 & 0.27 & -0.19 & 0.58 \\
\hline \multicolumn{11}{|l|}{ Calf, left } \\
\hline After 2 w & 0.29 & 0.01 & 0.02 & 0.79 & 0.12 & 0.12 & -0.28 & 0.00 & -0.22 & 0.00 \\
\hline $3 \mathrm{mo}$ & 0.03 & 0.73 & 0.05 & 0.73 & 0.06 & 0.70 & -0.07 & 0.52 & -0.25 & 0.05 \\
\hline \multicolumn{11}{|l|}{ Calf, right } \\
\hline After $2 \mathrm{w}$ & 0.36 & 0.00 & 0.09 & 0.26 & 0.16 & 0.01 & -.0 .28 & 0.00 & -0.026 & 0.00 \\
\hline $3 \mathrm{mo}$ & 0.13 & 0.25 & 0.13 & 0.27 & 0.18 & 0.24 & -0.25 & 0.02 & -0.23 & 0.02 \\
\hline
\end{tabular}

\begin{tabular}{|c|c|c|c|c|c|c|c|c|c|c|}
\hline & \multicolumn{2}{|l|}{$\mathrm{I}(20 \mathrm{~g} / \mathrm{l})$} & \multicolumn{2}{|l|}{ II $(40 \mathrm{~g} / \mathrm{l})$} & \multicolumn{2}{|l|}{ III (60 g/I) } & \multicolumn{2}{|l|}{ IV(tap) } & \multicolumn{2}{|l|}{ V (control) } \\
\hline & \begin{tabular}{|l|}
$\begin{array}{l}\text { Mean } \\
\text { difference } \\
(\mathrm{mm})\end{array}$ \\
\end{tabular} & $p$ & $\begin{array}{l}\text { Mean } \\
\text { difference } \\
(\mathrm{mm})\end{array}$ & $p$ & $\begin{array}{l}\text { Mean } \\
\text { difference } \\
(\mathrm{mm})\end{array}$ & $p$ & \begin{tabular}{|l|}
$\begin{array}{l}\text { Mean } \\
\text { difference } \\
(\mathrm{mm})\end{array}$ \\
\end{tabular} & $p$ & $\begin{array}{l}\text { Mean } \\
\text { difference } \\
(\mathrm{mm})\end{array}$ & $p$ \\
\hline \multicolumn{11}{|l|}{ Biceps } \\
\hline After $2 \mathrm{w}$ & 1.15 & 0.02 & 1.84 & 0.00 & 2.64 & 0.00 & -1.20 & 0.05 & -0.88 & 0.09 \\
\hline $3 \mathrm{mo}$ & 2.44 & 0.00 & 2.79 & 0.00 & 3.48 & 0.00 & 1.06 & 0.01 & 0.85 & 0.13 \\
\hline \multicolumn{11}{|l|}{ Triceps } \\
\hline After $2 \mathrm{w}$ & 1.28 & 0.00 & 1.79 & 0.00 & 2.32 & 0.00 & -1.11 & 0.05 & -0.56 & 0.44 \\
\hline $3 \mathrm{mo}$ & 2.88 & 0.00 & 2.58 & 0.00 & 3.43 & 0.00 & 1.86 & 0.00 & 1.50 & 0.02 \\
\hline \multicolumn{11}{|c|}{ Subscapular } \\
\hline After $2 \mathrm{w}$ & 1.33 & 0.00 & 1.47 & 0.00 & 2.43 & 0.00 & -0.94 & 0.01 & \begin{tabular}{|l|}
-0.18 \\
\end{tabular} & 0.84 \\
\hline $3 \mathrm{mo}$ & 2.20 & 0.00 & 2.00 & 0.00 & 2.52 & 0.00 & 0.26 & 0.55 & 1.18 & 0.09 \\
\hline \multicolumn{11}{|c|}{ Abdominal } \\
\hline After $2 \mathrm{w}$ & 0.76 & 0.01 & 1.95 & 0.00 & 3.14 & 0.00 & -1.03 & 0.08 & -0.53 & 0.20 \\
\hline $3 \mathrm{mo}$ & 1.58 & 0.00 & 2.14 & 0.00 & 3.07 & 0.00 & 1.28 & 0.04 & 0.76 & 0.09 \\
\hline \multicolumn{11}{|l|}{ Suprailiac } \\
\hline After $2 \mathrm{w}$ & 1.00 & 0.00 & 0.54 & 0.33 & 0.73 & 0.03 & -2.51 & 0.00 & 0.12 & 0.79 \\
\hline $3 \mathrm{mo}$ & 0.49 & 0.23 & 0.25 & 0.71 & 0.36 & 0.49 & -1.20 & 0.00 & 0.79 & 0.07 \\
\hline
\end{tabular}




\begin{tabular}{|c|c|c|c|c|c|c|c|c|c|c|}
\hline & \multicolumn{2}{|l|}{$\mathrm{I}(20 \mathrm{~g} / \mathrm{l})$} & \multicolumn{2}{|l|}{ II $(40 \mathrm{~g} / \mathrm{l})$} & \multicolumn{2}{|l|}{ III (60 g/l) } & \multicolumn{2}{|l|}{ IV(tap) } & \multicolumn{2}{|l|}{ V (control) } \\
\hline & \begin{tabular}{|l|}
$\begin{array}{l}\text { Mean } \\
\text { difference } \\
(\mathrm{mm})\end{array}$ \\
\end{tabular} & $p$ & \begin{tabular}{|l} 
Mean \\
difference \\
$(\mathrm{mm})$
\end{tabular} & $p$ & $\begin{array}{l}\text { Mean } \\
\text { difference } \\
(\mathrm{mm})\end{array}$ & $p$ & \begin{tabular}{|l|} 
Mean \\
difference \\
$(\mathrm{mm})$
\end{tabular} & $p$ & \begin{tabular}{|l} 
Mean \\
difference \\
$(\mathrm{mm})$
\end{tabular} & $p$ \\
\hline \multicolumn{11}{|l|}{ Thigh } \\
\hline After $2 \mathrm{w}$ & 1.37 & 0.02 & 2.41 & 0.00 & 4.50 & 0.00 & -1.54 & 0.03 & -2.58 & 0.00 \\
\hline $3 \mathrm{mo}$ & 2.76 & 0.000 & 3.67 & 0.00 & 5.16 & 0.00 & 0.97 & 0.06 & 1.65 & 0.06 \\
\hline \multicolumn{11}{|l|}{ Calf } \\
\hline After $2 \mathrm{w}$ & 1.65 & 0.00 & 4.04 & 0.00 & 3.29 & 0.00 & -2.37 & 0.00 & -2.29 & 0.00 \\
\hline $3 \mathrm{mo}$ & 3.69 & 0.00 & 5.23 & 0.00 & 4.77 & 0.00 & 0.48 & 0.47 & 1.62 & 0.07 \\
\hline
\end{tabular}

\begin{tabular}{|c|c|c|c|c|c|c|c|c|}
\hline Group/parameter & Mean & SD & $\overline{M D}$ & SD & Cl upper & Cl lower & $t$ & $p$ \\
\hline Cholesterol & 5.18 & 0.91 & \multirow{2}{*}{0.19} & \multirow{2}{*}{0.51} & \multirow{2}{*}{0.04} & \multirow{2}{*}{0.34} & \multirow{2}{*}{2.54} & \multirow{2}{*}{0.01} \\
\hline $\mathrm{I}$ & 4.99 & 0.96 & & & & & & \\
\hline \multirow[t]{2}{*}{ II } & 5.31 & 1.22 & \multirow{2}{*}{0.33} & \multirow{2}{*}{0.48} & \multirow{2}{*}{0.18} & \multirow{2}{*}{0.482} & \multirow{2}{*}{4.51} & \multirow{2}{*}{0.00} \\
\hline & 4.97 & 1.00 & & & & & & \\
\hline \multirow[t]{2}{*}{ III } & 5.70 & 1.32 & \multirow{2}{*}{-0.06} & \multirow{2}{*}{0.65} & \multirow{2}{*}{-0.25} & \multirow{2}{*}{0.14} & \multirow{2}{*}{-0.57} & \multirow{2}{*}{0.57} \\
\hline & 5.76 & 1.40 & & & & & & \\
\hline \multirow[t]{2}{*}{ IV } & 5.23 & 1.12 & \multirow{2}{*}{0.00} & \multirow{2}{*}{0.47} & \multirow{2}{*}{-0.16} & & & \\
\hline & 5.23 & 1.27 & & & & $0.1 /$ & $0.5 /$ & 0.95 \\
\hline V & 5.43 & 1.04 & 000 & 0.41 & -0.14 & 014 & בחס & 098 \\
\hline & 5.43 & 1.10 & 0.00 & 0.41 & -0.14 & 0.14 & 0.02 & 0.98 \\
\hline High density lipoprotein & 1.65 & 0.33 & ב000 & 019 & | & 003 & م8חת & בת10 \\
\hline 1 & 1.67 & 0.33 & -0.02 & 0.19 & -0.08 & 0.03 & -0.080 & 0.42 \\
\hline II & 1.65 & 0.32 & 017 & 021 & 011 & 0.23 & 5.45 & 0.00 \\
\hline & 1.48 & 0.29 & 0.11 & 0.21 & 0.11 & 0.23 & & \\
\hline III & 1.61 & 0.38 & 足 & Fרת & | & 0 & $1<0$ & 0 \\
\hline & 1.68 & 0.43 & -0.06 & 0.25 & -0.14 & 0.01 & -1.68 & 0.10 \\
\hline IV & 1.67 & 0.39 & 008 & 424 & مחת & 0163 & 179 & 8חم \\
\hline & 1.59 & 0.42 & 0.08 & 0.24 & 0.00 & 0.163 & $1 . / 9$ & 0.08 \\
\hline $\mathrm{V}$ & 1.50 & 0.26 & 014 & 025 & 003 & 024 & 264 & 001 \\
\hline & 1.37 & 0.28 & 0.14 & 0.25 & 0.03 & 0.24 & 2.64 & 0.01 \\
\hline Low density lipoprotein & 2.55 & 0.65 & | 10 & 039 & 2020 & בחת & 173 & רחת \\
\hline 1 & 2.65 & 0.74 & -0.10 & 0.35 & -0.22 & 0.02 & $\mid-1 . / 13$ & 0.05 \\
\hline II & 2.83 & 0.99 & 0.13 & 038 & 01 & 025 & 218 & 003 \\
\hline & 2.70 & 0.83 & & 0.30 & 0.01 & & 2.10 & \\
\hline III & 3.16 & 0.92 & مO & مدת & en & 016 & 071 & 018 \\
\hline & 3.12 & 0.95 & 0.04 & 0.40 & -0.08 & 0.16 & 0.71 & 0.48 \\
\hline IV & 2.77 & 0.94 & 003 & 035 & | & 016 & 055 & 058 \\
\hline & 2.73 & 1.05 & 0.03 & 0.35 & -0.09 & 0.16 & 0.55 & 0.38 \\
\hline $\mathrm{V}$ & 3.00 & 0.88 & 200 & 023 & | & & 0,15 & 065 \\
\hline & 2.98 & 0.91 & 0.02 & 0.25 & -0.08 & 0.12 & 0.45 & 0.65 \\
\hline Triglyceride & 1.05 & 0.58 & 012 & 032 & בחת & ב200 & 236 & בחת \\
\hline I & 0.93 & 0.52 & 0.12 & 0.35 & 0.02 & 0.22 & 2.30 & 0.02 \\
\hline II & 0.90 & 0.42 & 003 & م28 & -0.05 & |212 & 080 & 0.43 \\
\hline & 0.87 & 0.35 & 0.03 & & & & & \\
\hline III & 1.17 & 0.65 & 018 & 7 & 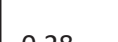 & | & 100 & Dro \\
\hline & 1.32 & 0.57 & -0.14 & 0.47 & -0.28 & 0.00 & -1.99 & 0.05 \\
\hline IV & 1.07 & 0.70 & 000 & 032 & | 12 & 011 & ב00 & 098 \\
\hline & 1.08 & 0.68 & 0.00 & 0.32 & -0.12 & 0.11 & -0.02 & 0.98 \\
\hline $\mathrm{V}$ & 1.05 & 0.53 & (1) & 060 & - 18 & 034 & 064 & 053 \\
\hline & 4.67 & 0.35 & & & & & & \\
\hline
\end{tabular}

Risks. No unintended effects were detected. 


\section{Ancillary analyses}

Significant after-treatment changes regarding cholesterol levels occurred in the geothermal and control groups. Positive changes in total cholesterol were seen after 20 (MD 0.2) and 40 $\mathrm{g} / \mathrm{I}$ (MD 0.3) baths; in Low Density Lipoprotein levels - after 40 $\mathrm{g} / \mathrm{l}$ baths (MD 0.1); in Triglyceride Levels - after $20 \mathrm{~g} / \mathrm{l}$ baths. There were significant negative changes in High Density Lipoprotein levels in the $40 \mathrm{~g} / \mathrm{l}$ and control groups, in Triglyceride Levels - in the $60 \mathrm{~g} / \mathrm{l}$ group (Table 6).

\section{Discussion}

\section{Limitations of the study}

The follow-up measurements were performed in the summer, when people are more physically active compared to other seasons. Thus, a longer follow-up period would be beneficial.

\section{Generalisability}

The results of the study demonstrated that 2 weeks of balneotherapy have a significant positive effect on body circumferences and skinfolds. The best short-term effect for reducing body $20 \mathrm{~g} / \mathrm{l}$ mineral water provides the best short-term effect for reducing body volume $(7.5 \mathrm{~cm})$, but the longest effect could be achieved using $60 \mathrm{~g} / \mathrm{l}$ geothermal water $(8.4 \mathrm{~cm}$ decrease from baseline). The greatest and longest-lasting decrease in skinfold was achieved after $60 \mathrm{~g} / \mathrm{l}$ procedures $(22.4 \mathrm{~mm}$ from baseline). No significant changes in BMI were found in the study groups. Concerning cholesterol, the most favourable were 20 (for total cholesterol and Triglyceride Levels) and $40 \mathrm{~g} / \mathrm{l}$ (for total cholesterol and Low Density Lipoprotein) mineral water baths.

\section{Interpretation}

However, the results of Suceveanu et al. demonstrated significant differences for LDL-cholesterol and HDL-cholesterol changes for patients with main cardiovascular risk factors who attended a rehabilitation programme with hydrotherapy included [16]. Mooventhan and Nivethitha suggest that hydrotherapy is widely used to improve immunity and for the management of hypercholesterolemia and add that there is a lack of evidences on the impact of water of different salinity and temperature [6]. The results of our study supplement the existing knowledge on the effect of the different mineralisation of water on cholesterol levels.

There are not many studies concerning balneotherapy on body volume or composition. There were reductions in limb vol- ume (average $0.8 \mathrm{~cm}, p<0.05$ ) and skin ridge thickness (average $2.2 \mathrm{~mm}, p<0,001$ ) after a 15-minute balneotherapy treatment earlier in the study [17]. 1 month after the treatment, the reduction in skin ridge $(p<0.05)$ and limb volume $(p<0.05)$ remained. After 4 months, a positive impact on the volume of the limbs was observed $(p<0.05)$, and the skin ridge was significantly lower than before the treatment $(p<0.01)$. It was found that a course of $20 \mathrm{~min} 27 \mathrm{~g} / \mathrm{I}$ geothermal water baths has the significant effect on BMI (average reduction of 0.5 ) and body fat percentage (2), with a positive effect until 4 month after treatment [17]. A study by Han et al. also found a significant reduction in BMI of $1.91 \mathrm{~kg} / \mathrm{m}^{2}$ after 3-week balneotherapy therapy compared at motivational dietary interview, which was only $0.20 \mathrm{~kg} /$ $/ \mathrm{m}^{2}$ after 1 year. This corresponds to a significant balneotherapy benefit of $1.71 \mathrm{~kg} / \mathrm{m}^{2}$ [18]. Our study did not show any positive changes, possibly because we used only a cooler mineral bath $\left(36^{\circ} \mathrm{C}\right.$ vs $\left.37^{\circ} \mathrm{C}\right)$ without other spa procedures or drinking mineral water, with no dietary recommendations, and the BMI of our participants was lower in the beginning (25-29 vs 31). A study by Kang Ki Yeon et al. on obese children shows that a hot spring bath could be an effective way of managing and treating obesity (BMI after 4 procedures lowered by 0.93 , as well as body fat mass by 2.28 and percentage of body fat by 4.04 [19]. The $\mathrm{Cl}-\mathrm{Na}-\mathrm{SO}_{4}-\mathrm{Ca}$ water we analysed produces effects that are specific to the main minerals. Of course, the chloride in the water improves cellular metabolism, tissue regeneration and blood and lymph circulation, as well as stimulates the organic and metabolic functions. In $34-36^{\circ} \mathrm{C}$ water, muscle tone decreases, peripheral arterial blood flow is stimulated, tissue trophic improves and swelling and pain lessen [13]. Data from researchers shows that spa therapy can modify plasma levels of the adipocytokines leptin and adiponectin [11], which is important for fat volumes in the body.

There is a need for further research for balneotherapy usage for integrated adiposity treatment and beauty therapy. A comparison of geothermal vs tap water treatment to clarify complex balneotherapy mechanisms for body composition changing with a standardised methodology, more respondents and a follow-up period are needed.

\section{Conclusions}

1. 20-minute baths of geothermal water are more effective than tap water for lowering of hypodermic fat content in the body and cholesterol level in serum.

2. $60 \mathrm{~g} / \mathrm{l}$ baths have a long-lasting effect on body fat volumes.

3. $20-40 \mathrm{~g} / \mathrm{l}$ geothermal water is appropriate for an antilipidemic effect.

Source of funding: This work was funded from the authors' own resources.

Conflicts of interest: The authors declare no conflicts of interest.

\section{References}

1. Moss GA. Water and health: a forgotten connection? Perspect Public Health 2010; 130(5), doi: 10.1177/1757913910379192.

2. Bouvier CE, Lieber J, Solimene U, et al. Proceedings of the European Thermal Meeting, Enghienles-Bains, France, 2013 [cited 15.05.2015]. Available from URL: http://www.europeanthermalmeeting.org.

3. Morer C, Roques CF, Françon A, et al. The role of mineral elements and other chemical compounds used in balneology: data from double-blind randomized clinical trial. Int J Biometeorol 2017; 61: 2159-2173, doi: 10.1007/s00484-017-1421-2.

4. Harris W. Balneotherapy overview [cited 15.05.2015]. Available from URL: https://health.howstuffworks.com/skin-care/beauty/skintreatments/balneotherapy.htm.

5. Stier-Jarmer M, Kus S, Frisch D, et al. Health resort medicine in non-musculoskeletal disorders: is there evidence of its effectiveness? Int J Biometeorol 2015; 59: 1523-1544, doi: 10.1007/s00484-015-0953-6.

6. Mooventhan A, Nivethitha L. Scientific evidence-based effects of hydrotherapy on various systems of the body. N Am J Med Sci 2014; 6: 199-209, doi: 10.4103/1947-2714.132935.

7. Pirogowicz I, Ornat M, Jerczak B, et al. The influence of physiotherapy on blood pressure in children and adolescents with respiratory tract diseases. Fam Med Prim Care Rev 2017; 19(1): 54-58, doi: https://doi.org/10.5114/fmpcr.2017.65092.

8. WHO. Obesity and overweight [cited 2.02.2018]. Available from URL: https://www.who.int/en/news-room/fact-sheets/detail/obesityand-overweight.

9. Kim TY, You SE, Ko YS. Association between Sasang constitutional types with obesity factors and sleep quality. Integr Med Res 2018; 7(4): 341-350, doi: 10.1016/j.imr.2018.06.007. 
10. Stephens JM, Argus CH, Driller MW. The relationship between body composition and thermal responses to hot and cold water immersion. J Hum Perform Extreme Environ 2014; 11(2): 1, doi: 10.7771/2327- 2937.1051.

11. Fioravanti A, Cantarini L, Bacarelli MR, et al. Effects of Spa therapy on serum leptin and adiponectin levels in patients with knee osteoarthritis. Rheumatol Int 2011; 31(7): 879-882.

12. Biro S, Masuda A, Kihara T, et al. Clinical implications of thermal therapy in lifestyle related diseases. Exp Biol Med 2003; 228(10): $1245-1249$.

13. Lithuanian Resort Research Center. Standardized Recommendation for Mineral Water Use in Resorts for Wellness, Prevention, Treatment and Rehabilitation. Druskininkai: Všl Lithuanian Resort Research Center; 2008.

14. International Society for the Advancement of Kinanthropometry. International Standards for Anthropometric Assessment (ISAK). Glasgow: International Society for the Advancement of Kinanthropometry; 2001.

15. Lawrenson D. How to measure your bofy fat proc. using calipers. 2012 [cited 6.01.2019]. Available from URL: https://www.muscleandstrength.com/tools/measure-bodyfat.

16. Suceveanu M, Pop D, Suceveanu P, et al. Effects of cardiovascular rehabilitation in patients admitted to the "Dr. Benedek Geza" hospital of rehabilitation in cardiovascular deseases, Covasna. Balneo Res J 2015; 6(1); 53-59, doi: 10.12680/balneo.2015.1086.

17. Rapolienè L. The effect of geothermal water on skin condition and body fat. Br J Med Med Res 2016; 18(10): 1-13.

18. Han C, Hahn AC, Fisher $\mathrm{Cl}$, et al. Women's facial attractiveness is related to their body mass index, but not their salivary cortisol. $A m$ J Hum Biol 2016; 28(3): 352-355.

19. Kang Ki Yeon, Ahn Taek Won, Han Jae Kyung. The Effect of Balneotherapy on Obesity Index and Body Composition on Obese Children. J Pediatr Korean Med 2013; 27(3): 29-40, doi: 10.7778/jpkm.2013.27.3.029.

Tables: 6

Figures: 3

References: 19

Received: 15.04 .2019

Reviewed: 29.04.2019

Accepted: 20.05 .2019

Address for correspondence:

Lolita Rapolienè, PhD, Assoc. Prof.

Klaipeda University

H. Manto 84

Klaipeda

Lithuania

Tel.: +370 69824342

E-mail: lolita.rapoliene@inbox.It 